\title{
Experimental comparison of control architectures
}

\author{
J.M. van de Mortel-Fronczak, J.P.M. Schmitz, J.E. Rooda \\ Eindhoven University of Technology, Department of \\ Mechanical Engineering, P.O. Box 513, 5600 MB Eindhoven \\ The Netherlands \\ Tel: +31402475697 \\ Fax: +31402452505 \\ e-mail: \{vdmortel,schmitz,rooda\}@senov.wtb.tue.nl
}

\begin{abstract}
Most control systems of flexible production cells have a hierarchical structure. They become very complicated and difficult to maintain and modify when the underlying production cells grow in size and complexity. Moreover, they are characterised by a relatively high sensitivity to failures. As opposed to that, heterarchical control systems are flexible, modular, easy to modify, and - to some extent - fault-tolerant. In this paper, the performance of two control systems, with a hierarchical and a heterarchical structure, based on an exemplary flexible production cell is evaluated by means of simulation experiments. Under ideal circumstances, both control systems perform equally well.
\end{abstract}

Keywords

Modelling, control architectures, simulation, performance evaluation 


\section{INTRODUCTION}

Flexible manufacturing systems are functionally organised according to the jobshop principle, meaning that departments are formed around particular types of equipment or operations. The manufacturing control system takes care of part handling and processing activities in order to transform materials into products. The main function of this control system is the scheduling of available resources. To handle the complexity, the control function can be distributed among several control components. According to (Chase and Aquilano, 1992), the scheduling function in a control system can be divided into releasing, allocating, sequencing and dispatching sub-functions. In this paper, only part allocation is discussed.

Control architectures structure the relations between the different control components, not only with regard to their positions, but also with regard to the way components interact with each other. The choice of suitable control architecture for a specific flexible production system is not self-evident and should carefully be considered. In (Dilts et al., 1993), four basic control architectures are discussed: centralised, hierarchical, hybrid and heterarchical. Most control systems of flexible production cells have a hierarchical structure characterised by modularity and fast response. They become very complicated and difficult to maintain and modify when the underlying production cells grow in size and complexity. Moreover, they are characterised by a relatively high sensitivity to failures. As opposed to that, heterarchical control systems are flexible, modular, easy to modify, and - to some extent - fault-tolerant. However, one should also be aware of disadvantages, such as: the absence of standards for communication protocols, often only local optimisation and high communication traffic.

In this paper, a quantitative comparison of the hierarchical and the heterarchical control architectures is discussed for a specific flexible manufacturing system.

According to the hierarchical architecture, the control function is decomposed into several modules that are arranged in a pyramid. Each control module is responsible for a small amount of information processing necessary to execute the control function. The modules in the pyramid obey master-slave principles.

According to the heterarchical control architecture, the control function is distributed among autonomous modules (agents) that negotiate with each other on equal terms. For negotiations, fixed communication protocols (Lin and Solberg, 1994) are used. Depending on the negotiation protocol and the speed of the communication network used, a time parameter plays an important role in the experiments. The influence of this parameter on the performance of the heterarchical control architecture is investigated.

The paper is structured as follows. In Section 2, two control models needed for the comparison are briefly discussed. Simulation results are discussed in Section 3. In Section 4, concluding remarks are presented. 


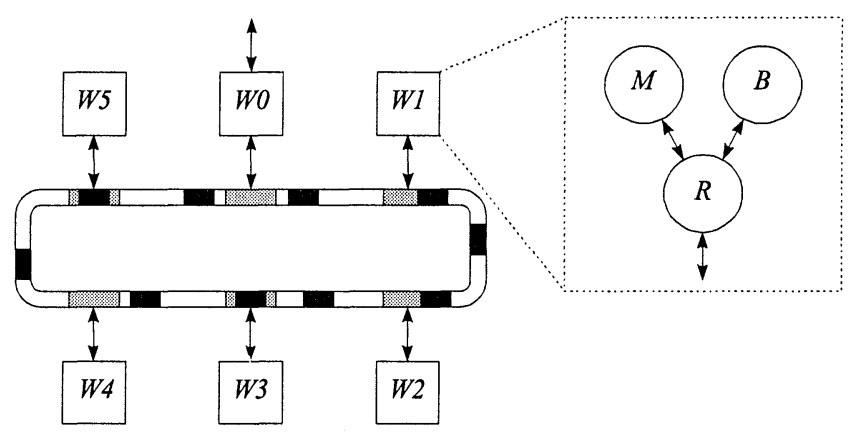

Figure 1 Flexible manufacturing cell

\section{TWO CONTROL MODELS}

For a quantitative comparison of control architectures, different models are needed, one for each control architecture. For a fair comparison, the models should only differ in exactly those aspects that must be compared. This means that the only differences apply to control components. Moreover, it is only possible to make a fair comparison if the rules on which the decision making is based, are the same in every model. This implies that although the architectures are different, the knowledge and decision rules used in the models must be the same. Consequently, the knowledge and decision rules used by the control components, must produce the same output if fed with the same input parameters. For example, both architectures must, according to its own structure and protocols, in the end select the same workstation. If this is not carefully done, not only control architectures, but also decision rules are compared.

In order to evaluate the control architectures in this context, several simulation experiments can be performed based on different modelling methods. As, nowadays, industrial systems consist of many components performing their actions in parallel, it seems justifiable to use for modelling a perception which exploits this parallel character, for instance, Petri nets (David and Alla, 1994) or Communicating Sequential Processes (Hoare, 1985). The models described according to the concepts borrowed from Communicating Sequential Processes form a starting point for simulation experiments described in this paper.

To perform experiments with control architectures, first a model of a flexible manufacturing cell is developed. In (Van de Mortel-Fronczak and Rooda, 1997), an abstract model of this cell is used to present a specification of a heterarchical control system. For simulation experiments described in this paper, a more detailed model (Schmitz, 1998) is used. 


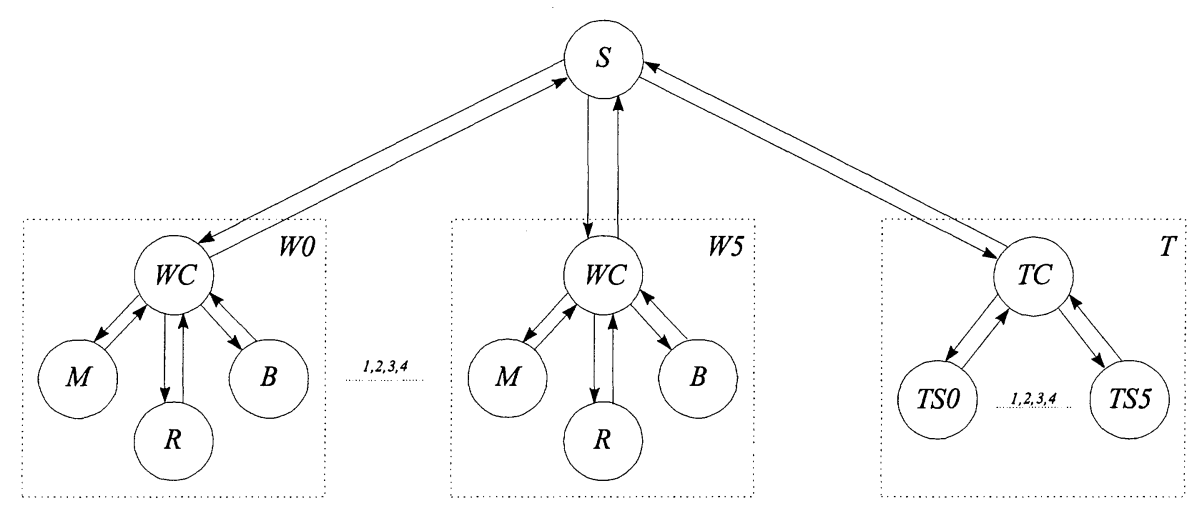

Figure 2 Hierarchical control architecture of the flexible manufacturing cell

The flexible manufacturing cell consists of six workstations (four machining workstations $W 1$ through $W 4$, one assembly station $W 5$, and one input-output station (WO) and an automatic transport system (Figure 1). The transport system consists of a conveyor and six conveyor stations (TSO through TS5) associated with workstations. The products are transported on pallets. Each workstation is equipped with a robot $(R)$ that moves products between the buffer $(B)$ and the machine $(M)$ inside the workstation. The same robot is used for moving products between the conveyor station and the buffer. The workstations can process different operations. Some operations can be performed on different workstations (parallel machines). Each machine can perform only one operation at a time on a product. An operation on a product cannot be performed until all the previous operations on the product are completed. The limiting resources are: the machine capacity, the processing time, the buffer space, the capacity and speed of the robot, the amount of pallets and the conveyor speed.

For this cell two models are developed, one for each control architecture. These models only differ in the components that take care of the control, viz. the allocating of the products to the workstations. In both architectures, the same decision rules are used to select a proper workstation for a product. First, the workstation has to be able to perform the requested operation. Next, the workstation with the lowest amount of products waiting for operation is selected.

In the hierarchical control system, the top level consists of the scheduler $S$ that takes care of allocating the products to the workstations (Figure 2). From the lower layer, the scheduler receives information about the system state. This information, and the information about the machines and the operations they can perform, is used to determine control tasks for the transport controller (TC) and for the workstation controllers $(W C)$, which form the second layer of control. The transport controller regulates the transportation of the parts and products to the right destination by instructing the transport stations. Each workstation controller takes care of the proper material handling within the corresponding workstation. 


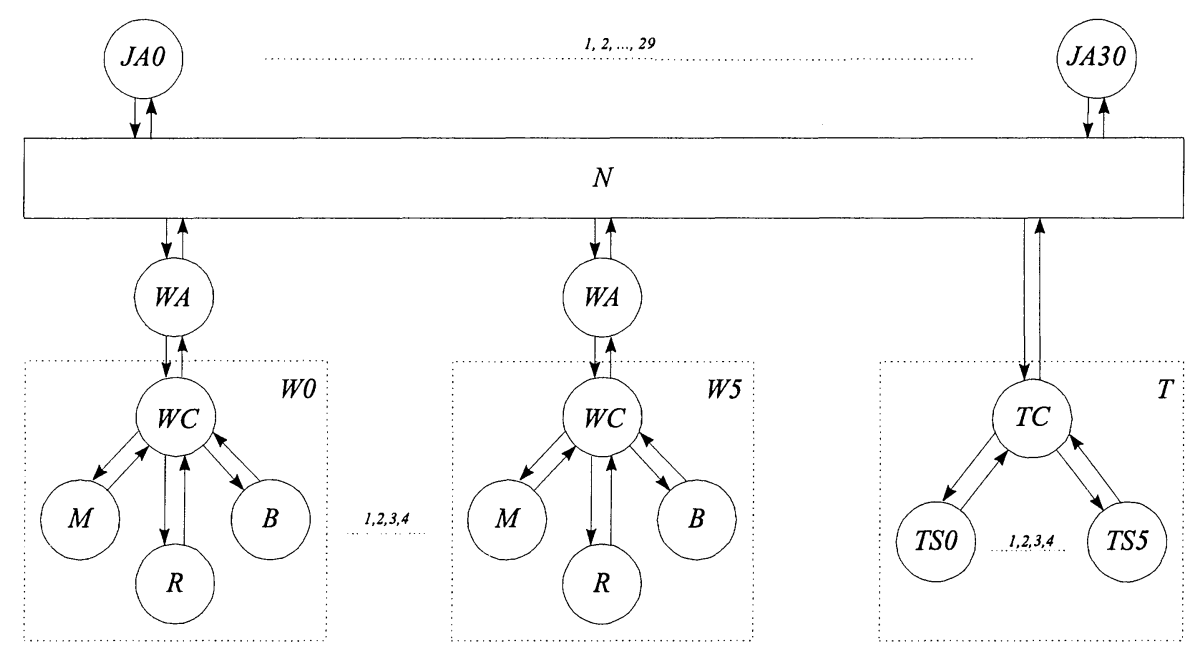

Figure 3 Heterarchical control architecture of the flexible manufacturing cell

In the heterarchical control system, job and workstation agents are used to take care of allocating products. Job agents $J A$ represent products that have to be processed, workstation agents $W A$ represent workstations in the manufacturing cell. Each job agent sends requests to all workstation agents for a subsequent operation to be performed. After a certain waiting time, the job agent selects a workstation depending on the replies from the workstation agents and the selection criterion (Lin and Solberg, 1994). The necessary communications take place via a network $(N)$ that connects all agents as shown in Figure 3.

\section{SIMULATION RESULTS}

To compare the performance of both control architectures, experiments are performed with two models briefly described in Section 2. As performance criteria for the comparison, the flow time $(\varphi)$, lateness $(L)$ and the number of tardy products $(n T)$ are chosen (Holthaus and Rajendran, 1997; Mahmoodi and Martin, 1997). The flow time is defined as the time between the moments at which a product enters and leaves the production system. The lateness is defined as the difference between the due date that was determined for a product, and the moment this product is finished. The number of tardy products is the amount of products that were unable to meet the due date.

An important parameter in the heterarchical control architecture is $t a$, modelling the deadline for bids of the workstation agents in response to requests from job agents. The presence of this deadline concept in the heterarchical architecture is relevant for fault-tolerance: if a workstation does not respond because of a break down, the remaining part of the control system can still function properly. 
Table 1. Experiments with hierarchical and heterarchical control architecture, $\varphi$ : average flow time (minutes); L: average lateness (minutes); nT: amount of tardy products

\begin{tabular}{|c|c|c|c|c|c|c|}
\hline \multirow[t]{2}{*}{ experiment } & \multicolumn{3}{|c|}{$\begin{array}{l}\text { hierarchical control } \\
\text { architecture }\end{array}$} & \multicolumn{3}{|c|}{$\begin{array}{l}\text { heterarchical control } \\
\text { architecture }\end{array}$} \\
\hline & $\varphi$ & $L$ & $n T$ & $\varphi$ & $L$ & $n T$ \\
\hline 1 & 124.79 & -48.71 & 20 & 125.12 & -48.48 & 22 \\
\hline 2 & 197.31 & 26.02 & 207 & 197.49 & 26.24 & 206 \\
\hline 3 & 124.11 & -47.83 & 219 & 124.72 & -47.24 & 253 \\
\hline 4 & 158.27 & -8.82 & 117 & 158.28 & -8.81 & 118 \\
\hline
\end{tabular}

Experiments with the deadline values $t a>0$ (experiments 1 through 3, Table 1), show that the performance of the model with hierarchical control is on average slightly better than the performance of the model with heterarchical control. This applies to the flow time, but also to the lateness and the amount of tardy products. The difference in performance is of the same order as the sum of the deadline values for the average amount of operations on a product. Moreover, this difference is many magnitudes smaller than the flow time. Experiment 4 (Table 1), with $t a=0$, shows that in this case, the performance indicators of both models are practically the same (Schmitz, 1998). This implies that the difference in performance is caused by setting the deadline on a value $t a>0$.

In Table 2, the parameters are given that are varied in the experiments listed in Table 1. These parameters are the number of experiments performed to determine the averages, the run length of each experiment (number of products), the work in process $(W I P)$ and the job-agent deadline $t a$ (minutes).

Moreover, additional output from simulation experiments (Schmitz, 1998) shows that both control systems take the same decisions.

Table 2. Parameters of the experiments

\begin{tabular}{|c|c|c|c|c|}
\hline experiment & \# runs & run length & $W I P$ & $t a$ \\
\hline 1 & 10 & 500 & 10 & $1 / 60$ \\
\hline 2 & 10 & 500 & 20 & $1 / 60$ \\
\hline 3 & 1 & 5000 & 10 & $1 / 60$ \\
\hline 4 & 10 & 500 & 15 & 0 \\
\hline
\end{tabular}




\section{CONCLUDING REMARKS}

In this paper, simulation experiments with two control architectures are presented that have been carried out in order to compare their performance indicators. Therefore, two models have been developed for the same flexible manufacturing cell, one with a hierarchical and one with a heterarchical control architecture. The models can easily be adapted or extended, both with respect to the number or configuration of workstations and with respect to the control functions. For a fair comparison, the models are constructed in such a way that the only differences are related to the relevant aspects. In the setting of this paper, this applies to the control components taking care of product allocation.

In the ideal situation, which means that no time delays occur in any control element of both control architectures, the performance of the hierarchical control architecture is the same as the performance of the heterarchical control architecture. This indicates that it does not matter how the information is distributed in the system if the control components are able to make proper use of it. Although both control architectures perform equally well, heterarchical control systems are still rather seldom used in automated manufacturing systems. This is probably due to the fact that the research in this area started only ten years ago, while the field of hierarchical control has been explored since the early ' 60 .

\section{REFERENCES}

Dilts, D.M., Boyd, N.P. and Whorms, H.H. (1993) The Evolution of Control Architectures for Automated Manufacturing Systems. Journal of Manufacturing Systems, 10 (1), 79 - 93.

Chase, R.B. and Aquilano, N.J. (1995) Productions \& Operations Management. A Life Cycle Approach. 7th edition, Irwin, Boston.

David, R. and Alla, H. (1994) Petri Nets for Modeling of Dynamic Systems - A Survey. Automatica, 30 (2), $175-202$.

Hoare, C.A.R. (1985) Communicating Sequential Processes. Prentice-Hall International, London.

Holthaus, O. and Rajendran, C. (1997) Efficient dispatching rules for scheduling in a job shop. International Journal of Production Economics, 48, 87 - 105.

Lin, Y.L. and Solberg, J. J. (1994) Autonomous control for open manufacturing systems, in Computer control of flexible manufacturing systems (eds. S.B. Joshi and J.S. Smith), Chapman and Hall, London.

Mahmoodi, F. and Martin, G.E. (1997) A new shop-based and predictive scheduling heuristic for cellular manufacturing. International Journal of Production Research, 35 (2), 313 - 326.

Schmitz, J.P.M. (1998) An experimental comparison of three control architectures for a CIM-cell. Technical Report 420185, Systems Engineering Group, Eindhoven University of Technology. The Netherlands. 
Van de Mortel-Fronczak, J.M. and Rooda, J.E. (1997) A case study in the design of control systems for flexible production cells. In Proceedings of MIM'97. Vienna, Austria, February 1997, 243 - 248.

\section{BIOGRAPHY}

Joanna M. van de Mortel-Fronczak graduated in computing science at the University of Mining and Metallurgy of Cracow in 1982. In 1993, she received the $\mathrm{Ph}$.D. degree in computing science from the Eindhoven University of Technology. Since then she works as a lecturer at the Department of Mechanical Engineering, Eindhoven University of Technology. Her research interests include specification, design, and verification of (machine) control systems.

Jos P.M. Schmitz is a graduate student at the Department of Mechanical Engineering, Eindhoven University of Technology. His technical interests include modelling and analysis of industrial systems.

Jacobus E. Rooda received the M.Sc. degree in food technology from the Wageningen Agricultural University in 1971. In 1978, he received the Ph.D. degree in mechanical engineering from the Twente University of Technology. Since 1985 he is a Professor of Systems Engineering at the Eindhoven University of Technology. His research interests include modelling of discrete-event and continuous-time systems, and the design of industrial systems. Prof. Rooda is a member of IEEE, IFIP-WG 5.7, IFAC (Mantech), ACM, and WEHTB (West European Professors of Technology Management). He also is a member of the editorial board of the International Journal of Advanced Manufacturing Technology. 